\title{
LIBERAÇÃO MIOFASCIAL E MANIPULAÇÃO ARTICULAR NO TRATAMENTO DE DORES TORÁCICAS INESPECÍFICAS
}

\author{
MYOFASCIAL RELEASE AND JOINT MANIPULATION \\ IN THE TREATMENT OF NONSPECIFIC DORSAL PAIN \\ Carla Albuquerque ${ }^{1}$, Ellen Vitória Samuelsson Mussulini ${ }^{1}$, \\ Paula Renata Olegini Vasconcellos ${ }^{2}$ e Gladson Ricardo Flor Bertolini ${ }^{3}$
}

\begin{abstract}
RESUMO
Objetivo: comparar as técnicas de manipulação articular e liberação miofascial de forma combinada e isolada em estudantes do ensino superior, com dor na coluna torácica. Materiais e métodos: foram analisados 40 indivíduos, distribuídos em quatro grupos: grupo controle, manipulação articular, liberação miofascial e somatória das técnicas. Todos os participantes foram avaliados em duas etapas: no primeiro contato com o participante ocorreu a Avaliação 1, e sete dias após os procedimentos a Avaliação 2. A avaliação da dor ocorreu com algômetro de pressão e dor ao frio com uso de pedra de gelo, ambos sobre dois pontos gatilho, também foi utilizado um questionário sobre crença de autoeficácia ao tratamento, uma Escala Visual Analógica e contabilizado o número total de disfunções vertebrais. Resultados: para todas as variáveis relacionadas à dor não houve redução do quadro nas diversas formas terapêuticas adotadas. Na análise de disfunções articulares, houve redução para os grupos em que foi utilizada a manipulação articular. Conclusão: a manipulação articular associada ou não a liberação miofascial reduziu o número de bloqueios articulares, contudo, não houve efeitos com respeito à dor.
\end{abstract}

Palavras-chave: Manipulações musculoesqueléticas, Pontos-gatilho, Coluna vertebral.

\section{ABSTRACT}

Objective: to compare the techniques of joint manipulation (MA) and myofascial release (LM) in combination and in isolation in higher education students, with pain in the thoracic spine. Materials and methods: Forty individuals were analyzed, distributed into four groups: control group, joint manipulation, myofascial release and sum of the techniques. All participants were evaluated in two stages: in the first contact with the participant, Evaluation 1 occurred, and seven days after the procedures, Evaluation 2. The evaluation of pain occurred with a pressure gauge and cold pain with the use of ice stone, both on two trigger points, it was also used a questionnaire on belief of self-efficacy to treatment, a Visual Analogue Scale as well as counting the total number of vertebral dysfunctions. Results: for all pain-related variables, there was no reduction in the various therapeutic forms adopted. In the analysis of joint dysfunctions, there was reduction for the groups in which joint manipulation was used. Conclusion: joint manipulation associated or not with myofascial release reduced the number of joint blocks; however, there were no effects with respect to pain.

Keywords: Musculoskeletal manipulations, Trigger points, Spine.

\footnotetext{
${ }^{1}$ Acadêmica do curso de fisioterapia da Universidade Estadual do Oeste do Paraná (Unioeste). E-mail: carlafmoto@ gmail.com; ellen.vi@hotmail.com

${ }^{2}$ Docente da graduação em fisioterapia da Unioeste. Mestre em Biociências e Saúde pela Unioeste. E-mail: paula. vasconcellos@hotmail.com

${ }^{3}$ Docente da graduação em fisioterapia e programa de pós-graduação em Biociências e Saúde - Unioeste. E-mail: gladsonricardo@gmail.com
} 


\section{INTRODUÇÃO}

A dor é uma experiência sensorial ou emocional desagradável e se torna crônica quando se prolonga por mais de três meses (RAUSCHKOLB; GOMES, 2016). Quando ocorre na coluna vertebral, pode ser classificada em específica ou inespecífica. Inespecífica quando ocorre sem uma doença ou lesão adjacente conhecida, e específica quando diagnosticada por profissionais da saúde como causada por doenças, por exemplo: infecção, tumor e hérnia de disco (MICHALEFF et al., 2012). A dor na coluna vertebral representa um dos principais problemas de saúde mundial que afeta a condição musculoesquelética, pode causar reflexos sociais e econômicos. Nos EUA $61 \%$ dos pacientes que procuram atendimento em serviços de saúde o fazem devido a problemas na coluna (DAGENAIS et al., 2015). No Brasil a procura é superada apenas pela hipertensão (THEME FILHA et al., 2015).

A dor na coluna torácica é a segunda mais referida por estudantes, em primeiro lugar vem a dor na cervical e em terceiro a dor na lombar (FERREIRA et al., 2015). Várias condições podem influenciar seu aparecimento, como o estresse, a má postura, a pressão biopsicossocial, o ambiente competitivo, a ansiedade e o estilo de vida; interferindo na saúde do estudante, prejudicando sua qualidade de vida e produtividade acadêmica (ALMEIDA; DUMITH, 2018; VEY; SILVA; LIMA, 2013).

Como tratamento existem terapias medicamentosas e complementares. Na procura de atendimentos de urgência, há uma maior utilização de fármacos, pois, é o padrão de se atenuar a dor, porém, muitas vezes o atendimento se torna generalizado. Terapias complementares tornam-se uma alternativa, visto que sua relação custo-benefício em comparação à medicina convencional é mais vantajosa ao paciente (DANTAS et al., 2014; FONSECA; LOPES; RAMOS, 2013). Dentre as formas complementares, a terapia manual tem sido frequentemente usada por fisioterapeutas, apresentando, dentro seus efeitos, redução dos níveis de dor (MICHALEFF et al., 2012; PRADO; GOUVEIA, 2014; RAUSCHKOLB; GOMES, 2016). Dentro da terapia manual, pode-se destacar duas técnicas: a manipulação articular (MA) e a liberação miofascial (LM) (ESTEVAM JUNIOR et al., 2015; RAUSCHKOLB; GOMES, 2016).

A MA, ou Thrust, é definida por um movimento de alta velocidade e baixa amplitude, respeitando o limite fisiológico e articular da anatomia humana. Essa técnica visa restabelecer o movimento funcional, e com isto diminuir a dor. Para escolher o local da aplicação da manipulação, deve-se considerar a disfunção individual do indivíduo (ESTEVAM JUNIOR et al., 2015; HUISMAN; SPEKSNIJDER; WIJER, 2013; RAUSCHKOLB; GOMES, 2016). Já a LM concentra-se em liberar tensões musculares, diminuindo o quadro álgico e aumentando a circulação sanguínea, dentre outras vantagens. Isto ocorre pela liberação de pontos gatilhos (PGs) e seus sintomas (SOUSA et al., 2015). Os PGs são causados por microlesões repetitivas e sobrecargas estáticas como alterações posturais, ou exagero de uso de certa região, também são conhecidos como Trigger Points, e são percebidos em 
forma de um nódulo palpável no exame físico (BATISTA; BORGES; WIBELINGER, 2012; PRADO; GOUVEIA, 2014).

Considerando a complexidade funcional da coluna vertebral, e que as algias provenientes dessa região são as principais causas de dor crônica na população, também levando-se em conta, a existência de poucos estudos sobre terapias manuais voltadas à dor musculoesquelética da coluna torácica (ESTEVAM JUNIOR et al., 2015; MARTIN et al., 2015), o objetivo desta pesquisa foi comparar as técnicas LM e MA de forma combinada e isolada em estudantes do ensino superior, com dor na coluna torácica.

\section{MATERIAIS E MÉTODOS}

Este ensaio caracteriza-se como clínico, intervencionista, aleatorizado, de caráter quantitativo. O estudo foi realizado no Centro de Reabilitação Física da Universidade Estadual do Oeste do Paraná (Unioeste), na cidade de Cascavel - PR, após a aprovação do Comitê de Ética em Pesquisa da Unioeste (parecer número 3.649.729).

A amostra foi composta por 40 acadêmicos, voluntários, independente de sexo e com idade variando entre 18 e 35 anos. O critério de inclusão foi: dor torácica de origem inespecífica presente por mais de três meses e os critérios de não inclusão: indivíduos com histórico de problemas cardíacos ou respiratórios; escoliose; cirurgia torácica recente; que estivessem em tratamento para dor, ou realizado nos últimos 06 meses. Como critério de exclusão: o comparecimento somente uma vez. A divisão foi feita por sorteio em urna, com nome escrito em papel.

Todos os participantes foram avaliados em duas etapas: no primeiro contato com o participante ocorreu a Avaliação 1 (AV1), e sete dias após os procedimentos, foi realizada a Avaliação 2 (AV2). Nas duas etapas as variáveis analisadas foram: dor por estímulo pressórico (algômetro de pressão) e térmico (gelo), questionário de autoeficácia (PSEQ), Escala Visual Analógica (EVA), número de disfunções e PGs encontrados. Foram contabilizados somente dois pontos gatilhos de avaliação para o presente estudo sendo um de cada lado da coluna torácica, escolhidos por palpação como os de maior dor, elegidos pelos indivíduos.

Para a avaliação do limiar de dor à pressão foi utilizado um algômetro da marca Kratos ${ }^{\circledR}$, modelo DDK-50 (São Paulo/Brasil), apresentando valores de pressão em gf. O voluntário permaneceu em decúbito ventral e a pressão foi efetuada por uma extremidade circular de 1,2 cm de diâmetro, nos locais previamente marcados com lápis dermográfico, em que o mesmo referiu dor, o estímulo pressórico ocorreu de forma gradativa até que o voluntário relatasse dor. Em seguida foi realizada a avaliação de dor ao frio, avaliando o limiar e intensidade desta, sempre sobre os dois PGs. Para tal, 
utilizou-se uma pedra de gelo posicionada diretamente sobre a pele, anotado o tempo (cronômetro) transcorrido até a informação de dor.

O Pain Self-efficacy Questionnaire (PSEQ) foi desenvolvido para avaliar a crença de autoeficácia ao tratamento para indivíduos com dor crônica. As opções de respostas variam de zero (nada confiante) a 6 (completamente confiante) para execução de uma tarefa. No final foi realizada a somatória das respostas das 10 questões (BONAFÉ; MARÔCO; CAMPOS, 2018). Também foi utilizada a EVA e solicitado que classificassem a intensidade da dor usando a numeração de " 0 a 10 ", em que " 0 " caracteriza ausência de dor e "10" dor insuportável.

A ficha de avaliação continha um esquema representando a região dorsal, no qual o pesquisador pode identificar os PGs, fazer anotações sobre o algômetro de pressão, o tempo cronometrado do gelo, quantas e quais vértebras estavam em disfunção (teste de Gillet, realizado com o apoio dos indicadores do terapeuta sobre os processos transversos vertebrais, analisando posicionamentos discrepantes, seguido por movimentação do tórax em flexão e extensão, analisando-se alterações posicionais da vértebra). Os indivíduos selecionados foram separados em quatro grupos: Grupo Controle (GC), Manipulação Articular (MA), Liberação Miofascial (LM) e as duas técnicas associadas (MA+LM), cada grupo com 10 participantes.

O grupo MA foi submetido a técnica de thrust. A manobra foi realizada posicionando o voluntário em decúbito dorsal, com os membros inferiores estendidos e os superiores cruzados sobre o tronco anteriormente. $\mathrm{O}$ terapeuta se posicionou contralateral à área a ser manipulada, sua mão cranial (baseado na posição paciente-terapeuta) foi apoiada sobre os cotovelos do voluntário, pressionando seu esterno nesta mão e o outro braço (caudal) envolveu o tórax do voluntário, com os dedos posicionados no nível da vértebra torácica em disfunção, então, foi solicitado uma inspiração e no final da expiração foi realizado um movimento brusco sobre os braços na parte central do tórax (SANTOS et al., 2015). Para o grupo LM foi utilizada a Técnica de Jones, que tem por finalidade desativação dos trigger points por meio da digitopressão, mantida por 90 segundos (PRADO; GOUVEIA, 2014).

Os dados foram analisados por Modelos Generalizados Mistos, com pós-teste LSD, em todos os casos o nível de significância foi de 5\%. Também foi avaliado o tamanho de efeito por d de Cohen, com base na primeira avaliação para determinado grupo, e classificado como: $<0,2$ : trivial; 0,2-0,5: pequeno; 0,5-0,8: moderado; >0,8: grande.

\section{RESULTADOS}

$\mathrm{Na}$ análise de dor pelo algômetro (Ponto 1 - P1, Ponto 2 - P2) e frio (P1, P2) verificou-se que ambas as formas de avaliação não apresentaram diferença significativa ( $p>0,05)$ entre os grupos, avaliações e interação dos fatores (grupo x avaliação). Para os tamanhos de efeito as variações foram de triviais a moderadas (Tabela 1$)$. 
Tabela 1 - Avaliação do quadro álgico pelo Algômetro (gf) e com o uso do gelo (segundos), bem como os tamanhos de efeito (TE) encontrados entre as avaliações (AV1 e AV2), nos diferentes pontos (P1 e P2). Grupos: controle (GC), manipulação articular (MA), liberação miofascial (LM) e associação de técnicas (MA+LM).

\begin{tabular}{|c|c|c|c|c|c|c|}
\hline \multirow{3}{*}{$\begin{array}{c}\begin{array}{c}\text { ALGÔME- } \\
\text { TRO }\end{array} \\
\text { GC }\end{array}$} & \multicolumn{2}{|c|}{ AV1 } & \multicolumn{4}{|c|}{ AV2 } \\
\hline & \multirow{2}{*}{$\begin{array}{c}\text { P1 } \\
4700 \pm 1665\end{array}$} & \multirow{2}{*}{$\begin{array}{c}\text { P2 } \\
5138 \pm 1578\end{array}$} & \multicolumn{2}{|c|}{ P1 } & \multicolumn{2}{|c|}{$\mathbf{P 2}$} \\
\hline & & & $5461 \pm 1500$ & $0,18 \mathrm{TE}$ & $4934 \pm 1978$ & $-0,11 \mathrm{TE}$ \\
\hline MA & $4843 \pm 1258$ & $5072 \pm 1481$ & $4688 \pm 947$ & $-0,13 \mathrm{TE}$ & $5616 \pm 1378$ & $0,36 \mathrm{TE}$ \\
\hline LM & $4193 \pm 1408$ & $4235 \pm 1624$ & $4329 \pm 1409$ & $0,09 \mathrm{TE}$ & $4599 \pm 1473$ & $0,22 \mathrm{TE}$ \\
\hline $\mathrm{MA}+\mathrm{LM}$ & $4330 \pm 1924$ & $4248 \pm 1942$ & $4718 \pm 1973$ & 0,19 TE & $5165 \pm 2326$ & $0,40 \mathrm{TE}$ \\
\hline \multirow[t]{2}{*}{ GELO } & \multicolumn{2}{|c|}{ AV1 } & \multicolumn{4}{|c|}{ AV2 } \\
\hline & $\mathbf{P 1}$ & $\mathbf{P 2}$ & \multicolumn{2}{|c|}{ P1 } & \multicolumn{2}{|c|}{$\mathbf{P 2}$} \\
\hline $\mathrm{GC}$ & $93 \pm 78$ & $69 \pm 46$ & $57 \pm 29$ & $-0,57 \mathrm{TE}$ & $52 \pm 19$ & $-0,45 \mathrm{TE}$ \\
\hline MA & $100 \pm 104$ & $91 \pm 64$ & $95 \pm 82$ & $-0,05 \mathrm{TE}$ & $108 \pm 80$ & $0,22 \mathrm{TE}$ \\
\hline LM & $114 \pm 42$ & $93 \pm 15$ & $126 \pm 96$ & $0,14 \mathrm{TE}$ & $105 \pm 67$ & $0,21 \mathrm{TE}$ \\
\hline $\mathrm{MA}+\mathrm{LM}$ & $58 \pm 24$ & $53 \pm 32$ & $70 \pm 46$ & $0,31 \mathrm{TE}$ & $60 \pm 43$ & $0,17 \mathrm{TE}$ \\
\hline
\end{tabular}

Quando analisadas as disfunções articulares, verificou-se que não houve diferença significativa entre os grupos $(p=0,613)$, mas houve entre as avaliações $(p<0,001)$, bem como interação entre os fatores $(p=0,001)$. Inicialmente havia mais disfunções no grupo associação, quando comparado ao controle; ao final apenas o grupo LM mostrou maior número ao comparar com os demais. Na comparação dentro dos grupos, houve redução para os grupos MA e associação (MA+LM). Os tamanhos de efeito foram grandes nos grupos em que houve manipulação (Tabela 2).

Tabela 2 - Avaliação das Disfunções Articulares, nos distintos momentos de avaliação (AV1 e AV2) e apresentação dos tamanhos de efeito (TE). Grupos: controle (GC), manipulação articular (MA), liberação miofascial (LM) e associação de técnicas (MA+LM).

\begin{tabular}{cccc}
\hline DISFUNÇÕES ARTICULARES & AV1 & AV2 & TE \\
\hline GC & $2,7 \pm 1,1 \mathrm{Aa}$ & $3,1 \pm 1,3 \mathrm{ABa}$ & 0,07 \\
MA & $3,8 \pm 0,9 \mathrm{Aba}$ & $2,3 \pm 1,3 \mathrm{Ab}$ & $-1,56$ \\
LM & $3,2 \pm 1,4 \mathrm{Aba}$ & $3,7 \pm 2,2 \mathrm{Ba}$ & 0,05 \\
MA+LM & $3,9 \pm 1,4 \mathrm{Ba}$ & $2,0 \pm 0,8 \mathrm{Ab}$ & $-1,82$ \\
\hline
\end{tabular}

Dados expressos em média e desvio-padrão. Letras maiúsculas iguais apresentam semelhança estatística entre os grupos, letras minúsculas entre as avaliações dentro dos grupos.

Ao examinar o questionário PSEQ, houve diferença significativa entre os grupos $(p=0,046)$, porém, não entre as avaliações $(p=0,986)$ e interações $(p=0,937)$, apontando que o grupo controle possuía piores escores (mais altos) do que MA e MA+LM; e LM apresentou escores intermediários. Os tamanhos de efeito foram triviais (Tabela 3). 
Tabela 3 - Avaliação pelo Pain Self-efficacy Questionnaire - PSEQ, nos distintos momentos de avaliação (AV1 e AV2) e tamanhos de efeito (TE). Grupos: controle (GC), manipulação articular (MA), liberação miofascial (LM) e associação de técnicas (MA+LM).

\begin{tabular}{cccc}
\hline PSEQ & AV1 & AV2 & TE \\
\hline GC A & $54,8 \pm 4,2$ & $54,8 \pm 3,9$ & 0,0 \\
MA B & $47,2 \pm 8,6$ & $48,5 \pm 10,5$ & 0,13 \\
LM AB & $50,9 \pm 5,4$ & $50,7 \pm 9,3$ & $-0,03$ \\
MA+LM B & $48,7 \pm 8,5$ & $47,5 \pm 13,5$ & 0,10 \\
\hline
\end{tabular}

Dados expressos em média e desvio padrão. Letras maiúsculas iguais apresentam semelhança estatística entre os grupos.

$\mathrm{Na}$ análise da EVA, verificou-se que houve diferença entre as avaliações ( $\mathrm{p}=0,004)$, mas não entre os grupos $(p=0,524)$, nem interação $(p=0,955)$, mostrando que houve redução da intensidade da dor entre a primeira e segunda avaliação. Os tamanhos de efeito foram moderados (tabela 4).

Tabela 4 - Avaliação pela Escala Visual Analógica - EVA, nos distintos momentos de avaliação (AV1 e AV2) e tamanhos de efeito (TE). Grupos: controle (GC), manipulação articular (MA), liberação miofascial (LM) e associação de técnicas (MA+LM).

\begin{tabular}{cccc}
\hline EVA & AV1 A & AV2 B & TE \\
\hline GC & $4,1 \pm 1,9$ & $3,3 \pm 1,9$ & $-0,53$ \\
MA & $4,4 \pm 2,7$ & $3,8 \pm 2,5$ & 0,49 \\
LM & $5,0 \pm 1,9$ & $4,0 \pm 1,9$ & 0,50 \\
MA+LM & $4,9 \pm 1,7$ & $3,3 \pm 2,1$ & $-0,79$ \\
\hline
\end{tabular}

Dados expressos em média e desvio padrão. Letras maiúsculas indicam diferenças significativas entre as avaliações.

\section{DISCUSSÃO}

A dor é de natureza subjetiva e pode ser influenciada por fatores sensoriais e afetivos, isso torna complexa sua investigação experimental. Contudo, na literatura são citados vários instrumentos de mensuração desse fenômeno sensitivo. Neste estudo foram utilizados quatro métodos de avaliação, sendo dois indutores de dor: o algômetro de pressão e a baixa temperatura; e dois de autoavaliação: o PSEQ e a EVA. Quando avaliada a hiperalgesia não foi possível notar diferenças significativas, tanto na indução ao frio, quanto à pressão. A algometria é um método objetivo de alta confiabilidade e a indução pelo frio é um teste com resultados que variam de confiável a excelente (PELEGRINI; VENANCIO; LIEBANO, 2012; ZEGALO et al., 2016). Contudo, pode-se sugerir que a percepção dolorosa de cada indivíduo pode ter afetado sua interpretação e os resultados. Além disso, deve-se considerar que ambas as formas avaliam alterações do limiar doloroso por indução de estímulo nocivo, ou seja, para tais métodos de avaliação as técnicas utilizadas não apresentaram efeito.

O PSEQ apresenta perguntas com relação às atividades de vida diária, portanto, analisa a função frente à dor, é um instrumento válido e confiável, sendo importante no estudo de conceitos 
envolvidos nas percepções da dor e autoeficácia (BONAFÉ; MARÔCO; CAMPOS, 2018). A EVA é de grande importância na mensuração da intensidade de dor, pois, é por meio dela que o paciente pode descrever ou ser avaliado quanto à exatidão de sua algia (BOTTEGA; FONTANA, 2010). Contudo, ambas as formas de avaliação não apresentaram diferenças significativas em favor de alguma terapia adotada, sendo que para PSEQ os tamanhos de efeito foram triviais, apontando que, caso tenha ocorrido, uma possível melhora da função não contribuiu para redução da dorsalgia nestes voluntários. O que foi comprovado pela EVA, apesar de terem ocorrido tamanhos de efeito moderados.

A MA tem por objetivo devolver a mobilidade articular, recuperando o movimento fisiológico, contribui assim com a melhora da função musculoesquelética (BOSCHI; LIMA, 2012). Neste estudo os grupos que receberam MA não obtiveram diferenças nas variáveis de dor analisadas. Tais resultados são opostos àqueles encontrados por Huisman, Speksnijder e Wijer (2013), que apresentaram eficácia na dor com tratamento de manipulação articular torácica, bem como a revisão de Rauschkolb e Gomes (2016), em que relatam diminuição do quadro álgico pela manipulação. Em estudo de caso, Graça (2016) avaliou o efeito agudo da MA torácica na dor lombar, relata diminuição do pico de ângulo da flexão anterior de tronco, assim como diminuição da EVA, durante o mesmo movimento, pré e pós-manipulação. No estudo de Boschi e Lima (2012) há relato que a manipulação torácica (T4) reduz a dor em repouso e durante o movimento ativo da coluna cervical.

A técnica de Jones quando aplicada isoladamente não apresentou diferença significativa em todas as variáveis ao ser comparada ao GC, o que contradiz a uma revisão sistemática (MARTINS; PEREIRA; FELÍCIO, 2019), em que os autores relatam benefícios sobre a dor e tensão muscular. O que também é citado por Ziani et al. (2017), que apontam benefícios da digitopressão sobre a dor em pessoas com fibromialgia.

Segundo Southerst et al. (2015), pacientes que recebem mais que uma modalidade de tratamento não medicamentoso para dor torácica de início recente, apresentam maior capacidade de recuperação, porém, este estudo comparou num período de apenas 7 dias. Santos et al. (2015) associaram técnica de thrust em uma vértebra torácica (T6) com a técnica de músculo energia de peitorais. A pesquisa mostrou que a associação de duas técnicas foi eficiente na melhora da mobilidade torácica e função pulmonar, no entanto, não houve grupo controle, nem se avaliou dor musculoesquelética. No presente estudo, a associação das duas técnicas, MA (técnica de thrust) com a LM (técnica de Jones), bem como apenas a MA, apresentaram redução significativa na quantidade de disfunções articulares de movimento, porém, ressalta-se que apesar de melhora no movimento vertebral, não houve a relação direta com a dor percebida. 


\section{CONCLUSÃO}

Conclui-se que a MA associada ou não a LM reduziu o número de bloqueios articulares, contudo, não houve efeitos com respeito à dor. Por fim, a literatura se mostrou escassa com relação ao tema, o que evidencia a importância desta e outras pesquisas correlacionadas. Recomenda-se que próximos estudos abordem um número maior de indivíduos, bem como maior número de intervenções, e analisar a dor a longo prazo para que se possa ter mais clareza sobre as terapias e suas comparações.

\section{REFERÊNCIAS}

ALMEIDA, L. M. DA S.; DUMITH, S. DE C. Association between musculoskeletal symptoms and perceived stress in public servants of a Federal University in the South of Brazil. Brazilian Journal of Pain, v. 1, n. 1, p. 9-14, 2018.

BATISTA, J. S.; BORGES, A. M.; WIBELINGER, L. M. Physical therapy treatment for miofascial pain syndrome and fibromyalgia. Revista Dor, v. 13, n. 2, p. 170-174, 2012.

BONAFÉ, F. S. S.; MARÔCO, J.; CAMPOS, J. A. D. B. Pain self-efficacy questionnaire and its use in samples with different pain duration time. Brazilian Journal Of Pain, v. 1, n. 1, p. 33-39, 2018.

BOSCHI, E. S.; LIMA, D. C. Efeitos da manipulação torácica na dor e amplitude de movimento da coluna cervical. Cippus - Revista de Iniciação Científica do UniLassalle, v. 1, n. 1, p. 78-91, 2012.

BOTTEGA, F. H.; FONTANA, R. T. A dor como quinto sinal vital: utilização da escala de avaliação por enfermeiros de um hospital geral. Texto \& Contexto - Enfermagen, v. 19, n. 2, p. 283-290, 2010.

DAGENAIS, S. et al. A systematic review comparing the costs of chiropractic care to other interventions for spine pain in the United States. BMC Health Services Research, v. 14, p. 474, 2015.

DANTAS, D. R. S. et al. Caracterização clínica dos pacientes com distúrbios musculoesqueléticos atendidos em um serviço público de reabilitação fisioterapêutica no município de São Francisco do Conde - Bahia. Revista de Ciências Médicas e Biológicas, v. 13, n. 2, p. 156-162, 2014.

ESTEVAM JUNIOR, J. B. A. et al. Efeitos da aplicação da técnica de thrust e liberação de ponto gatilho na amplitude de movimento da dorsiflexão em bailarinos. Revista Brasileira de Prescrição e Fisiologia do Exercício, v. 9, n. 55, p. 497-505, 2015. 
FERREIRA, T. C. D. R. et al. Relação de dores musculoesqueléticas, estresse e qualidade de vida em acadêmicos do último ano de fisioterapia de duas instituições de ensino superior. Revista da Universidade Vale do Rio Verde, v. 13, n. 1, p. 239-255, 2015.

FONSECA, J. C.; LOPES, M. J.; RAMOS, A. F. Pessoas com dor e necessidades de intervenção: revisão sistemática da literatura. Revista Brasileira de Enfermagem, v. 66, n. 5, p. 771-778, 2013.

GRAÇA, P. R. C. DA. Manipulação articular melhora dor e amplitude de movimento em paciente com dor lombar crônica: relato de caso. Corpus et Scientia, v. 12, n. 1, p. 5-12, 2016.

HUISMAN, P. A.; SPEKSNIJDER, C. M.; WIJER, A. DE. The effect of thoracic spine manipulation on pain and disability in patients with non-specific neck pain: a systematic review. Disability and Rehabilitation, v. 35, n. 20, p. 1677-1685, 2013.

MARTIN, A. R. et al. Acute pain from the perspective of minor trauma patients treated at the emergency unit. Revista Gaúcha de Enfermagem, v. 36, n. 2, p. 14-20, 2015.

MARTINS, A. P.; PEREIRA, K. P.; FELÍCIO, L. R. Evidence of myofascial release in the physiotherapeutic treatment: systematic review. Arquivos de Ciências do Esporte, v. 7, n. 1, p. 8-12, 2019. MICHALEFF, Z. A. et al. Spinal manipulation epidemiology: systematic review of cost effectiveness studies. Journal of Electromyography and Kinesiology, v. 22, n. 5, p. 655-662, 2012.

PELEGRINI, S.; VENANCIO, R. C.; LIEBANO, R. E. Efeitos local e sistêmico do laser de baixa potência no limiar de dor por pressão em indivíduos saudáveis. Fisioterapia e Pesquisa, v. 19, n. 4, p. 345-350, 2012.

PRADO, S. M. C.; GOUVEIA, G. P. DE M. Efeito da inibição muscular na funcionalidade do trapézio fibras superiores. Fisioterapia Brasil, v. 15, n. 3, p. 189-194, 2014.

RAUSCHKOLB, P.; GOMES, T. DO N. Efeitos das técnicas manuais de mobilização e manipulação articulares da coluna vertebral. Revista Saúde Integrada, v. 9, n. 17, p. 2-8, 2016.

SANTOS, J. J. A. DOS et al. Influência das técnicas de terapia manual osteopática na função respiratória. Arquivos de Ciências da Saúde da UNIPAR, v. 19, n. 3, p. 191-197, 2015. 
SOUSA, R. C. DE et al. Efeitos da liberação miofascial na qualidade e frequência da dor em mulheres com cefaleia do tipo tensional induzida por pontos-gatilho. Fisioterapia Brasil, v. 16, n. 3, p. 231-235, 2015.

SOUTHERST, D. et al. The effectiveness of noninvasive interventions for musculoskeletal thoracic spine and chest wall pain: a systematic review by the Ontario Protocol for Traffic Injury Management (OPTIMa) collaboration. Journal of Manipulative and Physiological Therapeutics, v. 38, n. 7, p. 521-531, 2015.

THEME FILHA, M. M. et al. Prevalence of chronic non-communicable diseases and association with self-rated health: National Health Survey, 2013. Revista Brasileira de Epidemiologia, v. 18, n. Suppl 2 , p. 83-96, 2015.

VEY, A. P. Z.; SILVA, A. C. DA; LIMA, F. S. T. DE. Análise de dor nas costas em estudantes de graduação. Disciplinarum Scientia. Série: Ciências da Saúde, v. 14, n. 2, p. 217-225, 2013.

ZEGALO, B. P. et al. Efeitos da estimulação elétrica nervosa transcutânea e da corrente de alta voltagem em indivíduos saudáveis. Saúde e Pesquisa, v. 9, n. 2, p. 291-297, 2016.

ZIANI, M. M. et al. Efeitos da terapia manual sobre a dor em mulheres com fibromialgia: uma revisão de literatura. Ciência \& Saúde, v. 10, n. 1, p. 48-55, 2017. 